\title{
Large scale production of photonic crystals on scintillators
}

\author{
Arno Knapitsch, Etiennette Auffray, Member, IEEE, George Barbastathis, Celine Chevalier, Chih-Hung Hsieh, \\ Jeong-Gil Kim, Shuai Li, Matthew S. J. Marshall, Radoslaw Mazurczyk, Pawel Modrzynski, Member, IEEE, \\ Vivek Nagarkar, Ioannis Papakonstantinou, Bipin Singh, Alaric Taylor, Paul Lecoq, Fellow, IEEE,
}

\begin{abstract}
Heavy inorganic scintillator based detectors are used in various applications. You can find them in high energy physics as well as in nuclear medical imaging systems but also in homeland security radiation monitoring devices. In all these different detectors, light is produced in the scintillator and has to be transported towards a photodetector. The standard optical coupling of such a detector suffers from an inefficient light extraction towards the photodetector due to the high index of refraction of the scintillator and the accompanying total internal reflections. With the means of photonic nano structuring of the different surfaces of the scintillator, the light transport can be optimized, which has a direct impact on the timing and light yield performance of the detector. Previous work from our group has already shown that photonic crystals (PhCs) can be used as diffraction gratings to improve the light coupling between a photodetector and a scintillator.Moreover, nanoscale surface structuring techniques could also be extended to the sidewalls, the wrapping, or the detector itself, which would open up a number of new possibilities for optimization of the light transport of scintillation based detectors. To show that PhCs can also be produced on a large industrial scale, we started to investigate different methods for cheap and large area PhC structuring. In this work, the current results on our efforts on PhC scintillator production will be described. The different projects include nanoimprint technologies, interference lithography and colloidal lithography. To conclude, we will summarize the different efforts of our group and collaborators and show up-to-date results of PhC improved scintillators.
\end{abstract}

Index Terms-photonic crystals, nano lithography, light extraction, scintillators, Monte-Carlo, light diffraction, nanoimprint, colloidal lithography.

\section{INTRODUCTION}

$\mathbf{P}$ HOTONIC crystal $(\mathrm{PhC})$ gratings have the potential to overcome the total internal reflection angle within scintillating crystals. This effect can significantly improve the coupling efficiency between high index of refraction scintillators and their detectors [1], [2]. The principle of the diffraction

Arno Knapitsch, Etiennette Auffray, Pawel Modrzynski and Paul Lecoq are with CERN, 1211 Geneva, Switzerland, e-mail: arno.knapitsch@cern.ch

Cline Chevalier and Radoslaw Mazurczyk are with the Universite de Lyon, Institut des Nanotechnologies de Lyon-INL, UMR CNRS 5270 Ecole Centrale de Lyon, F-69134 Ecully, France.

George Barbastathis, Chih-Hung Hsieh, Shuai Li and Jeong-Gil Kim are with the Dept. of Mech. Eng., Massachusetts Inst. of Technol., Cambridge, MA 02139, USA

Vivek Nagarkar, Matthew S. J. Marshall and Bipin Singh are with Radiation Monitoring Devices Inc. (RMD), MA 02472, USA

Ioannis Papakonstantinou and Alaric Taylor are with the Department of Electronic and Electrical Engineering, University College London, WC1E 7JE, U.K

This work was carried out in the frame of the Crystal Clear Collaboration effect can be seen in Figure 1. Different Bloch modes, which are propagating in the periodic nano structured interface, are able to diffract certain modes into the ambient material [3]. Previous samples of photonic crystal grating covered scintillators were produced by a deposition of an auxiliary layer of silicon nitride on the extraction face of the scintillator pixel. This can be achieved by adaptation of reactive sputtering deposition, spin-coating and electron beam lithography (EBL) parameters [4]. Our collaboration could successfully produce several PhC slabs on top of $1.2 \times 2.6 \times 5.0 \mathrm{~mm}^{3}$ lutetium oxyorthosilicate (LSO) scintillators. The scanning electron microscope images of the $\mathrm{PhC}$ pattern of these scintillator samples can be seen in Figure 2 and the corresponding measurements in Table I. The manufacturing of PhC samples using EBL is rather slow (a few $\mathrm{mm}^{2} /$ day) and therefore hardly suitable for large scale production. Therefore, we investigate techniques to speed up the process and to produce large $\mathrm{PhC}$ pattern covered scintillators at a reasonable cost.
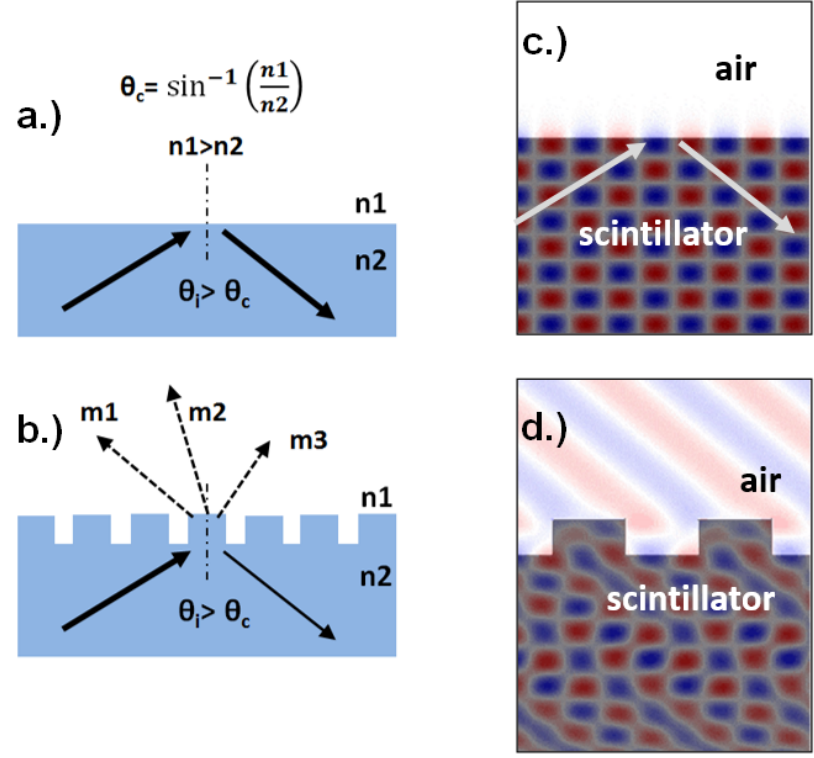

Fig. 1. Photons with an impinging angle larger than the critical angle $\theta_{c}$ cannot escape the crystal (a). Photonic crystal induced diffraction supports the extraction of certain modes $\left(m_{-2}, m_{-1}, m_{1}, m_{2}, \ldots\right)$ of otherwise reflected photons (b). The EM field plot to the right shows the difference of a flat air and LYSO interface (c) compared to a nano structured interface (d) in our CAMFR simulation tool [5] when a plane wave above the critical angle is impinging the interface. 


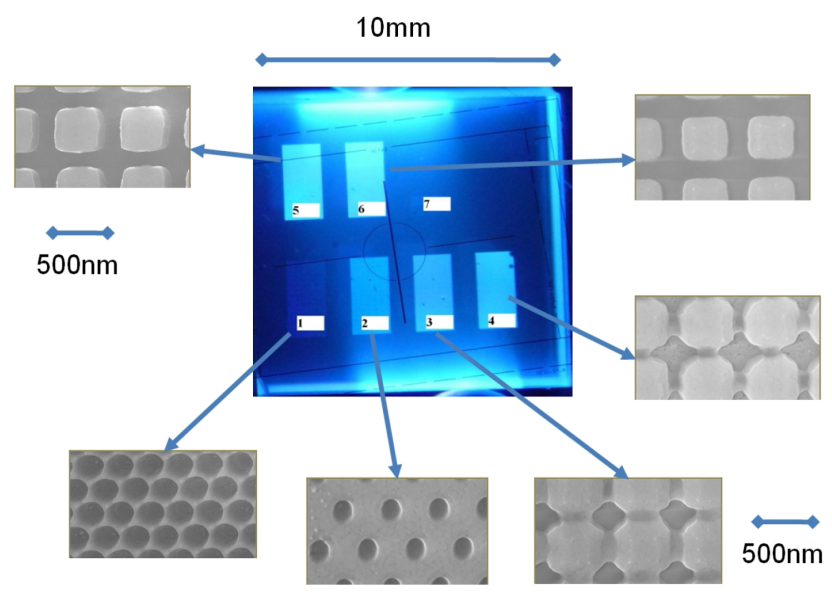

Fig. 2. Scanning electron microscope pictures of the six different $\mathrm{PhC}$ patterns produced on top of the $10 \mathrm{~mm} \times 10 \mathrm{~mm} \times 5 \mathrm{~mm}$ large base LSO crystal [3].

TABLE I

MeAn VALUes of THE LIGHT YIELD $[\mathrm{Pht} / \mathrm{MeV}]$ OF THE DifFerent PHC SAMPLES MEASURED WITHOUT WRAPPING AND WITHOUT OPTICAL GLUE [2]. THE GAIN IS CALCULATED AS A RATIO BETWEEN THE LIGHT YIELD OF THE PHC CRYSTAL AND THE REFERENCE CRYSTAL (SEE COLUMN P7-REF).

\begin{tabular}{lccccc}
\hline \hline & p7-ref & p2 & p3 & p4 & p6 \\
\hline Light Yield [Pht/Mev] & 2168 & 2279 & 2841 & 2815 & 3383 \\
Gain & 1.0 & 1.05 & 1.31 & 1.3 & 1.56 \\
\hline \hline
\end{tabular}

\section{Hybrid Photonic CRYstal PATterns}

In contrast to the simple $\mathrm{PhC}$ structure of holes or rods in our previous work [3], we propose hybrid photonic crystal patterns for an even better extraction efficiency [6] [7] [8]. Additionally, a cone shaped structure could potentially have better mechanical properties for the imprint process. Hybrid photonic crystal patterns combine two concepts, a conical shape to reduce Fresnel reflection of the zero order at a wide range of impinging angles, whereas the diffractive structure is meant to extract light beyond the critical angle (see Figure 3 and 4). The combination is difficult since the first structure (reducing Fresnel reflection) requires a sub-wavelength pitch, whereas the second structure (diffraction of otherwise totally reflected light) needs to be of a diffractive nature with a period bigger than the wavelength. Fortunately, if the period is near the emission wavelength, a mix of both effects can be observed [9] [10] [11] and by fine-tuning the different parameters of the structure we can balance them effectively to maximize light extraction. Examples of patterns which combine these two effects can be the cone structure (see Figure 4) or the regular $\mathrm{PhC}$ pattern covered with sub-wavelength cones (see Figure 8). Another idea would be to cover the classical diffraction grating with several thin layers of gradually ascending index of refraction. This would have similar anti reflection properties than the sub-wavelength nano cones. The simulation of the efficiencies of these structures was realized using a framework consisting of a PhC simulation software (CAMFR [5]) and a Monte-Carlo light ray tracing software (SLITRANI [12]). The model could be verified by our light yield and angular
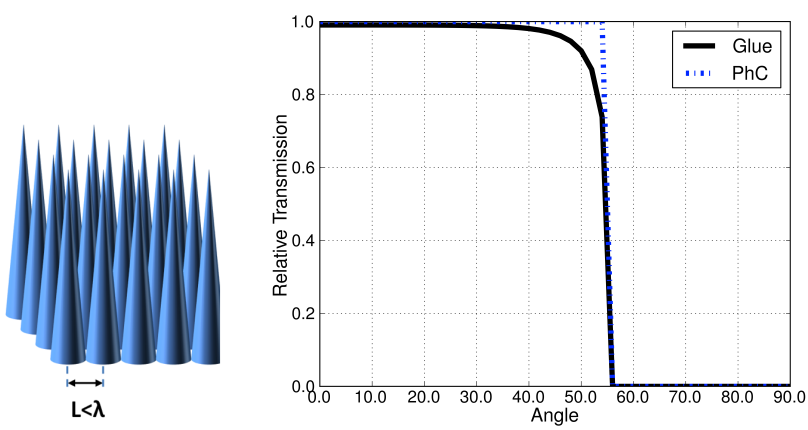

a.)
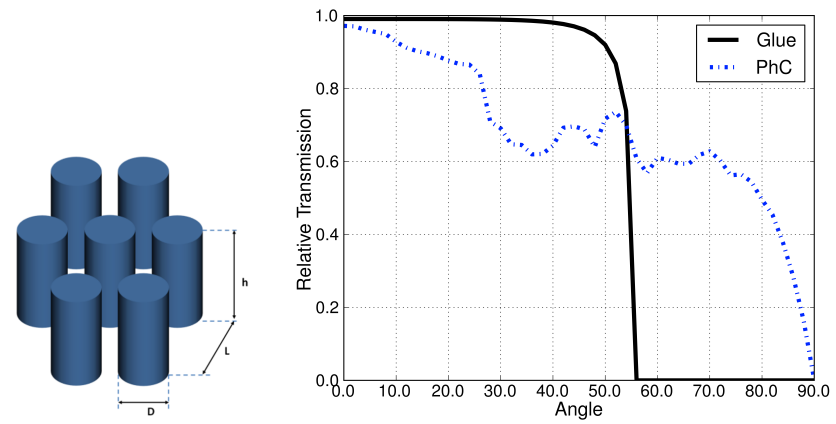

b.)

Fig. 3. Purely sub-wavelength conical anti reflection structure (a) to eliminate Fresnel reflections and wavelength scale diffractive structures (b) which can overcome the total internal reflection as shown in the transmission chart to the right of it.

distribution measurements [3] and also by independent timing measurements [13]. The transmission simulated for an optimized hybrid $\mathrm{PhC}$ grating like shown in Figure 4 produces a different gain for different types of scintillator crystals. The reason for that is manly the scintillator aspect ratio and the type of wrapping, which both have a big influence on the angular distribution of photons impinging on the $\mathrm{PhC}$ structure. If the $\mathrm{PhC}$ structure is made out of $\mathrm{TiO}_{2}$ like simulated (index of refraction $=2.5)$ and if it is applied to an $10 \times 10 \times 10 \mathrm{~mm}^{3}$ LYSO crystal, the simulation framework predicts a gain of $30 \%$ compared to a optically glued unstructured reference sample. The light extraction gain is still $20 \%$ when the index of refraction of the $\mathrm{PhC}$ material is 2.0 .

\section{NANOIMPRINTING}

Nanoimprinting is a fast and cheap way to reproduce a nano scaled pattern on a large area. In Figure 5, the four basic steps of the imprint procedure can be seen. A polymer on top of a high index of refraction material (silicon nitride $\mathrm{Si}_{3} \mathrm{~N}_{4}$ or titanium dioxide $\mathrm{TiO}_{2}$ ) is deposited on one side of a scintillator which is then imprinted with the PhC pattern. The stamp can be reused which makes the method feasible for the patterning of large areas. Afterwards, the imprinted pattern is etched in the high index material using reactive ion etching which results into the final $\mathrm{PhC}$ pattern. The technique can also be used to directly imprint the PhC material instead of just producing a mask. First results of a $\mathrm{PhC}$ pattern made by imprint technology in a high index of refraction polymer 

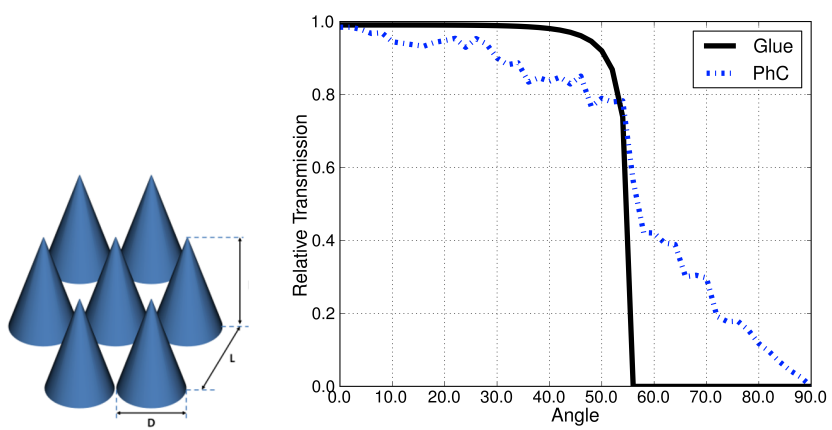

Fig. 4. Hybrid photonic crystal structure as a combination of sub-wavelength and diffractive structures (see Figure 3). If the cone period and size is comparable to the wavelength of the light, the cones partly act as diffraction grating in addition to their gradient index matching nature. The simulation parameters for this transmission simulation were set to a cone period of $700 \mathrm{~nm}$ and a height of $1.5 \mu \mathrm{m}$. The index of refraction of the cones was set to 2.5 and they were completely immersed into an optical glue of 1.5 index of refraction as an optical coupling medium to the detector. The simulation was computed at a wavelength of $420 u \mathrm{~m}$ which is the peak emission wavelength of LYSO.

which is on top of a LYSO scintillator can be seen in Figure 6. (a)

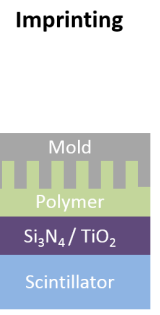

(b)

Demolding
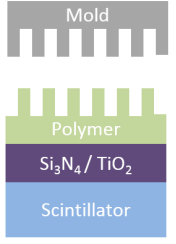

(c)

Etching
Result
Fig. 5. The four basic steps of the nanoimprint method. Imprinting in a polymer resist (a), hardening and demolding (b), etching (c) and the final cleaning of remaining polymer resist (d). (a)
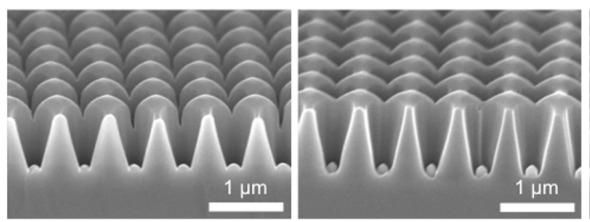

(c)

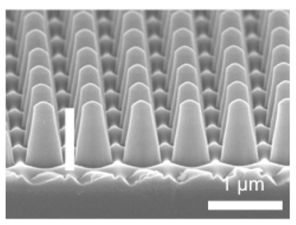

Fig. 6. Nanoimprint of three different $\mathrm{PhC}$ patterns in high index of refraction polymers on top of a silicon slab. Pictures with the courtesy of Radiation monitoring devices (RMD).

\section{Colloidal Lithography}

Nanospheres of different materials and different diameters are available on the market. They can be used to produce large areas of PhC patterns by self organization. The results of one of the techniques is shown in Figure 7 (LangmuirBlodgett process), where we used mono-disperse polystyrene (PS) spheres of $400 \mathrm{~nm}$ diameter dispersed in ethanol. The colloidal solution was then gently deposited into a purified water bath. Upon evaporation of the solvent, the nanospheres formed a highly ordered floating monolayer over the entire area. The resulting floating nanospheres were then compressed by a barrier to a highly-packed monolayer of hexagonal symmetry and then transferred onto the receiving substrate by dipcoating. By using $\mathrm{O} 2$ plasma treatment, the nanospheres were controllably shrunk $(7, b)$. Evaporation of a thin Cr layer and subsequent lift-off resulted in a hard mask being left behind on the substrate resembling a periodic nanohole array $(7, c)$ which can be used for reactive ion etching of the subjacent layer. By using high index of refraction materials (e.g. $\mathrm{TiO}_{2}$ ) the spheres can also be used as a diffraction grating itself which would be a very cost effective patterning approach since no further treatment would be necessary (compare similar aproach using PS nanospheres [14]). (a)

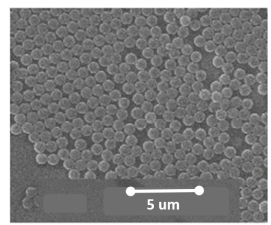

(b)

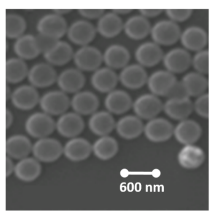

(c)

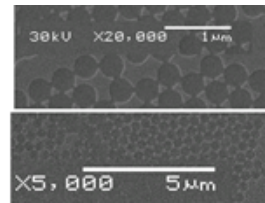

Fig. 7. Photonic mask production in a chromium layer by colloidal lithography. The polystyrene (PS) nano spheres after dip-coating are shown in a, the same sample after the PS nano spheres have been treated with $\mathrm{O} 2$ plasma can be seen in $b$ and $c$ shows a chromium layer which is deposited after the shrinking and the removal of the PS spheres (liftoff).

\section{REsUlts}

\section{A. Hybrid Structures}

By combining the anti-reflective properties of a cone structure with the diffractive properties of hole structures (see Figure 8) the light extraction efficiency can be further optimized [6] [8] [7]. The pattern shown in these SEM pictures was uniformly produced on a $10 \mathrm{~mm}$ diameter silicon slab and the patterning of an appropriate LYSO slab with this technique is currently investigated.

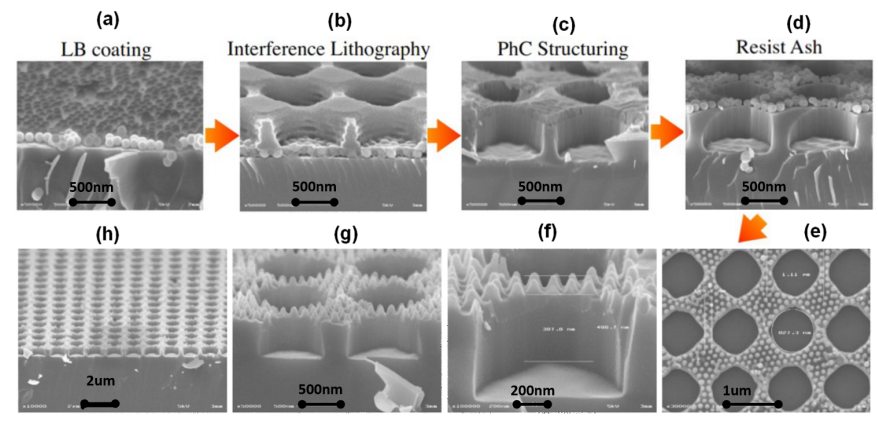

Fig. 8. Manufacturing of a hybrid PhC structure in silicon to be used as an imprint stamp. The process involves a combination of several different lithography steps including self-assembly and interference lithography techniques.

\section{B. Nanoimprint Structures}

Using a high index of refraction polymer (1.68 index of refraction) and an off-the-shelf conical nanoimprint stamp we 
produced several PhC structured LYSO scintillator cubes of the dimensions of $10 \times 10 \times 10 \mathrm{~mm}^{2}$. The cubes showed a uniform and well developed imprint result on more than $90 \%$ of the surface (see SEM image of Figure 9). When using an optical glue with an index of refraction of 1.5 for the coupling of the scintillator to the PMT, the refractive index contrast is rather small and therefore the $\mathrm{PhC}$ gain is theoretically limited to $5-10 \%$. But since the imprinted $\mathrm{PhC}$ pattern was of good quality and uniformity and the absorption of the polymer was negligible, we have already measured a gain of $5 \%$ for those samples when comparing it to a unstructured and glue coupled reference sample of the same type. Higher gain is expected with new polymer formulas which are currently under development.
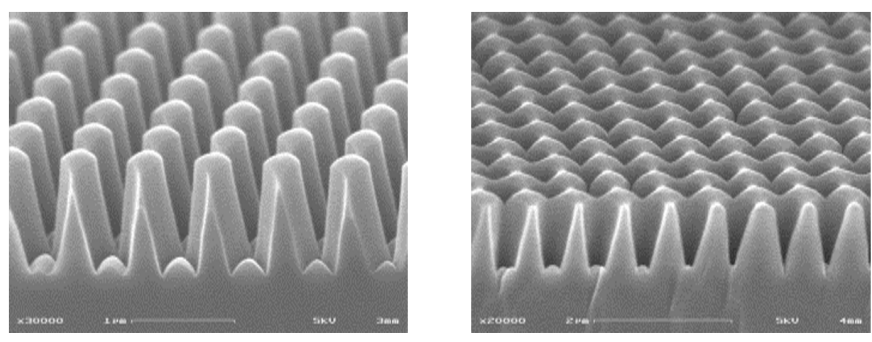

Fig. 9. Two different samples of a nanoimprint of an off-the-shelf nano cone pattern in a high index of refraction polymer (1.68 index of refraction).

\section{CONCLUSION}

Small scintillator pixels covered with $\mathrm{PhC}$ gratings were produced as a demonstrator already in 2011 by our group, and our collaborators [2]. The research in this field continues, and at the moment efforts are focused onto two main projects which are shown in this work. High index of refraction materials that can be deposited on top of a scintillator, and can also be shaped using state of the art nano patterning techniques have to be found and investigated. Our simulations show, that the diffractive nature of a $\mathrm{PhC}$ structure clearly improves with the index of refraction contrast between the two materials used in the pattern [3], therefore a transparent and high index of refraction material is needed. Second, techniques to reproduce $\mathrm{PhC}$ patterns on a large scale and with reasonable effort are investigated together with some new designs of the diffractive gratings (nano cones and hybrid structures). At the moment we have successfully produced titanium dioxide $\mathrm{TiO}_{2}$ and silicone nitride $\mathrm{Si}_{3} \mathrm{~N}_{4}$ layers on top of different scintillators. As shown in the results of this work, we have started to produce $\mathrm{PhC}$ patterns on scintillators using different large scale patterning techniques. With the current polymer imprint samples having a patterned area of $10 \times 10 \mathrm{~mm}^{2}$, a gain of 5\% compared to un-patterned reference sample could be observed. Our simulations indicate that the gain is expected to reach up to $20 \%$ when the index of refraction of the $\mathrm{PhC}$ material will be increased above 2.0 for the direct polymer imprints and 30\% for the RIE etched substrates of an index of refraction close to 2.4 or above.

\section{ACKNOWLEDGMENT}

This work was supported by the U.S. Department of Homeland Security, Domestic Nuclear Detection Office, under the competitively awarded contract HSHQDC-13-C-B0040. This support does not constitute an express or implied endorsement on the part of the government.

Additionally the work was supported by a Marie Curie Early Initial Training Network Fellowship of the European Community's seventh Framework Program under contract number (PITN-GA-2011-289355-PicoSEC-MCNet) and the EU funded ERC Advanced Grant N338953-TICAL.

\section{REFERENCES}

[1] M. Kronberger, E. Auffray, and P. Lecoq, "Probing the concepts of photonic crystals on scintillating materials," Nuclear Science, IEEE Transactions on, vol. 55, no. 3, pp. 1102 -1106, june 2008.

[2] A. Knapitsch, E. Auffray, C. W. Fabjan, J.-L. Leclercq, P. Lecoq, X. Letartre, and C. Seassal, "Photonic crystals: A novel approach to enhance the light output of scintillation based detectors," Nuclear Instruments and Methods in Physics Research Section A: Accelerators, Spectrometers, Detectors and Associated Equipment, vol. 628, no. 1, pp. 385-388, 2011. [Online]. Available: http: //linkinghub.elsevier.com/retrieve/pii/S0168900210015494

[3] A. Knapitsch and P. Lecoq, "Review on photonic crystal coatings for scintillators," International Journal of Modern Physics A, vol. 29, no. 30, p. 1430070, 2014. [Online]. Available: http: //www.worldscientific.com/doi/abs/10.1142/S0217751X14300701

[4] A. Knapitsch, E. Auffray, C. Fabjan, J.-L. Leclercq, X. Letartre, R. Mazurczyk, and P. Lecoq, "Results of photonic crystal enhanced light extraction on heavy inorganic scintillators," Nuclear Science, IEEE Transactions on, vol. 59, no. 5, pp. 2334-2339, Oct 2012.

[5] P. Bienstman, "Rigorous and efficient modelling of wavelength scale photonic components," Ph.D. dissertation, Universiteit Gent, 2001. [Online]. Available: http://photonics.intec.ugent.be/download/phd $\backslash_{\text {_t }} 104$. pdf

[6] C.-H. Chang, J. A. Dominguez-Caballero, H. J. Choi, and G. Barbastathis, "Nanostructured gradient-index antireflection diffractive optics," Opt. Lett., vol. 36, no. 12, pp. 2354-2356, Jun 2011. [Online]. Available: http://ol.osa.org/abstract.cfm?URI=ol-36-12-2354

[7] J. Kim, C. Hsieh, J. Gardener, B. Singh, A. Knapitsch, and G. Barbastathis, "Conical photonic crystals for enhancing light extraction efficiency from high index of refraction materials," Optics Express, vol. in review, 2015.

[8] C.-H. Hsieh, "Design and manufacturing of all-dielectric optical metamaterial with gradient index of refraction," Ph.D. dissertation, Massachusetts Institute of Technology, 2015.

[9] R. Magnusson and T. K. Gaylord, "Diffraction regimes of transmission gratings," J. Opt. Soc. Am., vol. 68, no. 6, p. 809, Jun 1978. [Online]. Available: http://www.osapublishing.org/abstract.cfm?URI= josa-68-6-809

[10] M. G. Moharam and T. K. Gaylord, "Diffraction analysis of dielectric surface-relief gratings," J. Opt. Soc. Am., vol. 72, no. 10, pp. 1385-1392, Oct 1982. [Online]. Available: http://www.osapublishing. org/abstract.cfm?URI=josa-72-10-1385

[11] M. G. Moharam and L. Young, "Criterion for bragg and raman-nath diffraction regimes," Appl. Opt., vol. 17, no. 11, pp. 1757-1759, Jun 1978. [Online]. Available: http://ao.osa.org/abstract.cfm?URI= ao-17-11-1757

[12] F.-X. Gentit, "Litrani: a general purpose monte-carlo program simulating light propagation in isotropic or anisotropic media," Nuclear Instruments and Methods in Physics Research Section A: Accelerators, Spectrometers, Detectors and Associated Equipment, vol. 486, no. 12, pp. $35-39,2002$, proceedings of the 6th International Conference on Inorganic Scin tillators and their Use in Scientific and Industrial Applications. [Online]. Available: http://www.sciencedirect.com/science/article/pii/S016890020200671X

[13] S. Gundacker, E. Auffray, B. Frisch, P. Jarron, A. Knapitsch, T. Meyer, M. Pizzichemi, and P. Lecoq, "Time of flight positron emission tomography towards 100ps resolution with $1(\mathrm{y})$ so: an experimental and theoretical analysis," Journal of Instrumentation, vol. 8, no. 07, p. P07014, 2013. [Online]. Available: http://stacks.iop.org/1748-0221/8/i= $07 / \mathrm{a}=\mathrm{P} 07014$ 
[14] Z. Zhu, B. Liu, C. Cheng, H. Chen, M. Gu, Y. Yi, and R. Mao, "Broadband light output enhancement for scintillator using whispering-gallery modes in nanospheres," physica status solidi (a), vol. 211, no. 7, pp. 1583-1588, 2014. [Online]. Available: http://dx.doi.org/10.1002/pssa.201431032 